\title{
Enhancement of radiation sensitivity in lung cancer cells by celastrol is mediated by inhibition of $\mathrm{Hsp90}$
}

\author{
JI-HYUN LEE ${ }^{1,3^{*}}$, KYU JIN CHOI ${ }^{1 *}$, WOO DUCK SEO ${ }^{2,4}$, SOON YOUNG JANG ${ }^{1}$, MIRA KIM ${ }^{1}$, BYONG WON LEE ${ }^{4}$, \\ JUN YOUNG KIM ${ }^{4}$, SEONGMAN KANG ${ }^{3}$, KI HUN PARK ${ }^{5}$, YUN-SIL LEE ${ }^{2}$ and SANGWOO BAE ${ }^{1}$ \\ Laboratories of ${ }^{1}$ Modulation of Radiobiological Responses and ${ }^{2}$ Radiation Damage Control, Korea Institute of \\ Radiological and Medical Sciences (KIRAMS), Seoul 139-706; ${ }^{3}$ School of Life Sciences and Biotechnology, \\ Korea University, Seoul 136-701; ${ }^{4}$ Department of Functional Crop, National Institute of Crop Science (NICS), \\ Rural Development Administration (RDA), Miryang 627-803; ${ }^{5}$ Division of Applied Life Science (BK21 program), \\ EB-NCRC, Institute of Agriculture and Life Science, Gyeongsang National University, Jinju 660-701, Republic of Korea
}

Received November 1, 2010; Accepted December 29, 2010

DOI: 10.3892/ijmm.2011.601

\begin{abstract}
The radiosensitizing activity of celastrol, a quinone methide triterpene was examined. We found that celastrol treatment of the NCI-H460 lung cancer cell line increased radiation-induced cell killing. The increased radiosensitivity was correlated with decreased levels of Hsp90 clients, such as EGFR, ErbB2 and survivin as well as with increased p53 expression. Celastrol inhibited the ATP-binding activity of Hsp90. Furthermore, celastrol treatment dissociated an Hsp90 client protein, EGFR, and this in turn resulted in degradation of the client protein. These results were not observed with another structurally similar triterpenoid, $6 \beta$-acetonyl-22 $\beta$ hydroxytingenol (TG), suggesting that a specific structural feature of the triterpenoid is required for radiosensitization. Moreover celastrol treatment increased p53 levels by phosphorylating Ser15 and Ser20 residues as well as by inhibiting its proteasomal degradation. Celastrol may be considered an effective radiosensitizer acting as an inhibitor of $\mathrm{Hsp} 90$ and a p53 activator. The two activities could be applicable to a broad range of cancer cells with either wild-type or mutant p53 because either activity could be effective for the enhancement of radiation cell killing. Further analysis with other triterpenoids should identify the functional moiety of the structure and additional candidates for effective radiosensitizers, which can be used in combined radiotherapy.
\end{abstract}

Correspondence to: Dr Sangwoo Bae, Laboratory of Modulation of Radiobiological Responses, Korea Institute of Radiological and Medical Sciences (KIRAMS), 75 Nowon-gil, Nowon-gu, Seoul 139-706, Republic of Korea

E-mail: swbae@kcch.re.kr

${ }^{*}$ Contributed equally

Key words: radiation sensitivity, celastrol, Hsp90, client proteins, p53

\section{Introduction}

Cellular sensitivity or resistance to ionizing radiation is determined by multiple processes that are involved in the cell cycle, proliferation, apoptosis, DNA repair and signal transduction. Agents that target these processes may modify radiation sensitivity leading to either enhanced or decreased cell killing (1). Given the diverse processes and molecules that are associated with radiation responses, targeting of multiple regulators, instead of only one factor, should substantially modify radiosensitivity (2). Thus, identification of such agents that are able to target multiple factors for radiosensitization is of importance.

Celastrol is a triterpenoid that can affect diverse cellular processes. Celastrol has been associated with antiinflammation, anticancer and treatment of Alzheimer's disease with activities such as Hsp90 inhibition, proteasome inhibition and modulation of gene expression (3). For instance, celastrol suppresses the proinflammatory cytokine-induced $\mathrm{NF}-\kappa \mathrm{B}$ activation via inhibition of $\mathrm{I} \kappa \mathrm{B} \alpha$ kinase resulting in decreased expression of $\mathrm{NF}-\kappa \mathrm{B}$ target genes such as IAP, Bcl2, Bcl-xL, COX-2 and survivin $(4,5)$. As those genes are mainly involved in cell protection from stress conditions, combined treatment with celastrol and therapeutic drugs such as TNF- $\alpha$ or TRAIL potentiates anticancer capabilities $(4,6)$. Inhibition of the Hsp90 chaperoning machinery is another mechanism for the anticancer activity of celastrol. Celastrol inhibits Hsp90 ATPase activity and disrupts the Hsp90-Cdc37 complex formation which plays a crucial role in the maturation of various kinases (7). Disruption of Hsp90-Cdc37 client kinases results in degradation of these kinases and consequent inactivation of the enzymes. As many of the Hsp90 clients, which include Raf1, Akt, and EGFR (epidermal growth factor receptor) (8), are associated with cellular proliferation and tumor resistance to anti-cancer treatment, the ability of celastrol as a kinase destabilizer has been exploited in cancer cells (9). A potent proteasomal inhibitory activity of celastrol has also been reported (10). Celastrol inhibits the chymotrypsin-like activity of the purified proteasome and celastrol treatment of prostate 
cancer cells results in accumulation of ubiquitinated proteins and induction of apoptosis (10).

Considering the diverse celastrol target molecules and their roles in radiation responses, we examined the effects of celastrol on radiation sensitivity. For this purpose, we purified natural celastrol and another structurally similar triterpenoid compound, $6 \beta$-acetonyl-22 $\beta$-hydroxytingenol (TG) and compared their effects on radiation sensitivity. Our comparison revealed that celastrol has a radiosensitizing effect, whereas a structurally very similar compound, TG, does not. Our results show that the radiosensitizing effect of a specific triterpenoid is correlated with the expression levels of key modulators of cellular radiation responses.

\section{Materials and methods}

Extraction, isolation and identification of celastrol and $T G$. Stem roots $(5 \mathrm{~kg})$ of Tripterygium regelii were airdried, chopped and extracted three times with $95 \% \mathrm{MeOH}$ (3x10 liters) for 7 days at room temperature. The combined extract was concentrated, and the dark residue (453 g) was partitioned between water and chloroform (1:1 liter). The organic layer was washed with brine, dried over anhydrous $\mathrm{Na}_{2} \mathrm{SO}_{4}$, and concentrated to give a dark brown residue $(310 \mathrm{~g})$ which was subjected to chromatography on a silica gel column. The extract $(310 \mathrm{~g})$ was suspended in $\mathrm{H}_{2} \mathrm{O}(2 \times 1$ liters $)$ and partitioned with organic solvents $\left(\mathrm{CHCl}_{3}, \mathrm{BuOH}\right)$ of different polarities to yield $\mathrm{CHCl}_{3}(162 \mathrm{~g}), \mathrm{BuOH}(125 \mathrm{~g})$, and $\mathrm{H}_{2} \mathrm{O}$ $(150 \mathrm{~g})$ extracts. The $\mathrm{CHCl}_{3}$ and $\mathrm{BuOH}$ extracts were subjected to column chromatography using a silica gel with a hexaneacetone gradient and a hexane-EtOAc gradient. The $\mathrm{CHCl}_{3}$ extract (162 g) was subjected to column chromatography (glass column 10x80 cm) using a silica gel $(1.5 \mathrm{~kg} ; 70-230$ mesh; Merck, NJ, USA), eluted with gradient mixtures of hexane (4 liters) and hexane-acetone, of increasing polarity $(30 / 1 \rightarrow 1 / 6)$, and finally with $\mathrm{MeOH}$. Fifteen pooled fractions (fractions 1-30) were obtained after combining fractions with similar TLC profiles from this initial column chromatography. The column was eluted with solvents of increasing polarity $\left(\mathrm{CHCl}_{3}\right.$-acetone) to give 30 fractions. Fraction 5 (14.3 g) was chromatographed on a silica gel column with hexane-acetone to give 45 fractions (5.1-5.30). The fractions from 5.11-5.18 and 5.21-32.27 were evaporated to give celastrol (1080 mg) [Rf, 0.53 (hexane/acetone $=4 / 1)]$ and TG $(32 \mathrm{mg})[\mathrm{Rf}, 0.45$ $\left(\mathrm{CHCl}_{3} /\right.$ acetone $\left.\left.=4 / 1\right)\right]$, respectively. The structures of isolated celastrol and TG were confirmed by spectroscopic analysis and comparison with previously reported values (11). ${ }^{1} \mathrm{H}$ - and ${ }^{13} \mathrm{C}-\mathrm{NMR}$ at 500 and $125 \mathrm{MHz}$, respectively, and 2D-NMR data were obtained on a Bruker AM-500 spectrometer in $\mathrm{CDCl}_{3}$. The structural information on celastrol and TG is available upon request.

Cell lines and treatment. The human lung cancer cell line NCI-H460 was obtained from the American Type Culture Collection (Manassas, VA, USA). The cell line was grown in RPMI-1640 (Life Technologies, Inc., Rockville, MD, USA) containing $5 \mathrm{mM}$ glutamate and $10 \%$ fetal bovine serum (Invitrogen, Carlsbad, CA, USA) and maintained at $37^{\circ} \mathrm{C}$ in an atmosphere of $5 \% \mathrm{CO}_{2}$ and $95 \%$ room air. Cell cultures were irradiated using a ${ }^{137} \mathrm{Cs}$ gamma-ray source (Atomic
$\mathbf{A}$

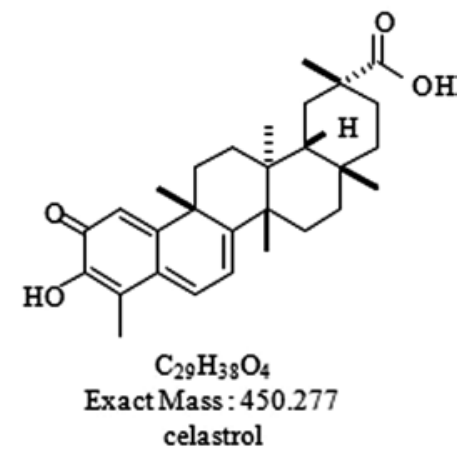

B

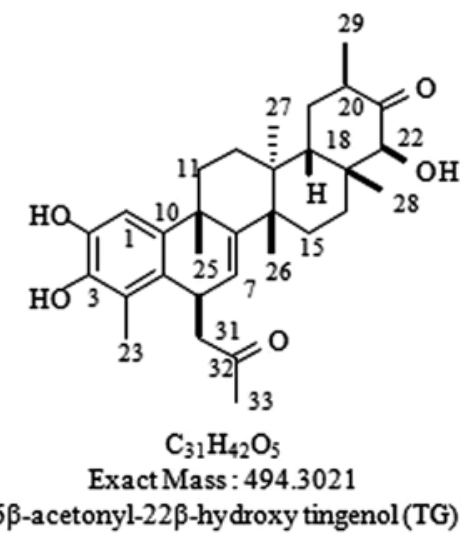

Figure 1. The chemical structure of (A) celastrol and (B) $6 \beta$-acetonyl-22 $\beta$ hydroxytingenol (TG).

Energy of Canada Ltd., Mississauga, Canada) at a dose rate of $3.81 \mathrm{~Gy} / \mathrm{min}$. Cell proliferation was measured by the MTT assay (Sigma, MO, USA) following the manufacturer's instructions. The colony forming ability after the treatment was compared using a clonogenic assay as previously described (12).

Immunoblot analysis, immunoprecipitation, and ATP-pull down assay. Cells were scraped into PBS, centrifuged, and the cell pellet was resuspended in 3 volumes of lysis buffer [40 mM Tris- $\mathrm{HCl}$ (pH 8.0), $120 \mathrm{mM} \mathrm{NaCl}, 0.1 \%$ Nonidet $\mathrm{P}-40$, and protease inhibitors] and proteins were extracted. Immunoblot analysis and immunoprecipitation were performed as previously described (13). The antibodies to Hsp90, Akt, EGFR, ErbB2, p53, MDM2, p27, Bax, Cyclin D1, Bcl2 and survivin were obtained from Santa Cruz Biotechnology (Santa Cruz, CA, USA), and antibodies to $\beta$-actin were from Sigma. p53 phospho-antibody to Ser15 and Ser20 were from Cell Signaling Technology (Beverly, MA, USA). Blots were developed by peroxidase-conjugated secondary antibody and proteins were visualized by ECL procedures (Amersham Biosciences, Piscataway, NJ, USA) according to the manufacturer's protocol. The ATP-pull down assay was performed using the ATP-Binders resin from Novagen (Madison, WI, USA) following published procedures (9).

\section{Results}

We isolated celastrol and TG from T. regelii to test their effect on radiation sensitivity (Fig. 1). Treatment of NCI-H460 lung 
A

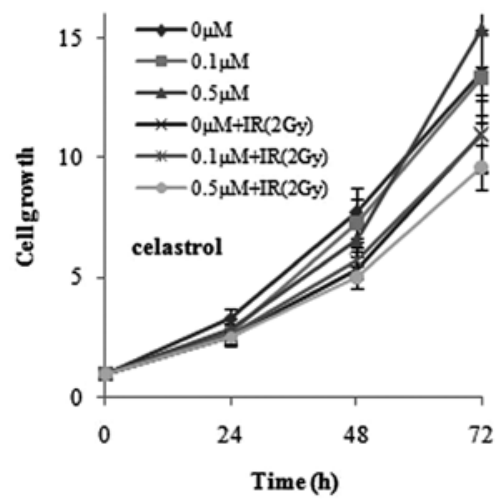

C

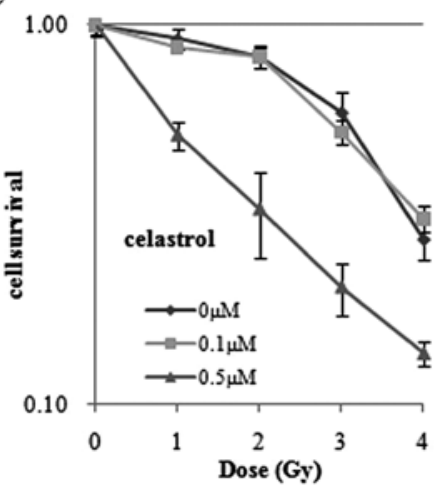

B

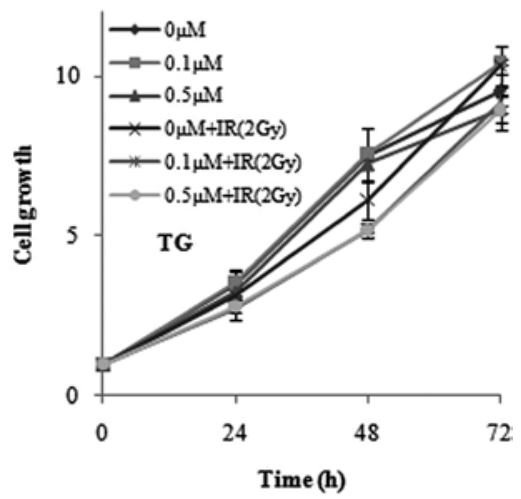

D

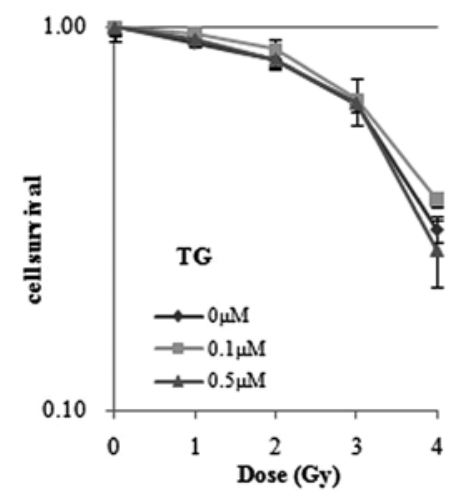

Figure 2. Effect of celastrol and TG on cell growth and clonogenic survival after irradiation. NCI-H460 cells were treated with Celastrol (A) or TG (B) and cell growth was measured by the MTT assay. Cells were incubated with $0,0.1$ and $0.5 \mu \mathrm{M}$ of celastrol or TG for 24,48 and $72 \mathrm{~h}$ with or without ionizing radiation (IR). Clonogenic cell survival was measured after treating cells with celastrol (C) or TG (D) in the presence or absence of IR. IR doses of $0,1,2,3$, and 4 Gy were used for irradiation.

cancer cells with celastrol alone exhibited a moderate growth inhibitory effect as shown by the decreased cell growth after 2 or 3 days of incubation (Fig. 2A). TG treatment also decreased cell proliferation but the decrease was less than that of celastrol (Fig. 2B). The clonogenic survival assay revealed that irradiation of the lung cancer cells together with celastrol enhanced radiation sensitivity by decreasing clonogenic cell survival (Fig. 2C). Cell treatment with $0.5 \mu \mathrm{M}$ celastrol enhanced the radiation sensitivity, whereas, celastrol concentrations up to $0.1 \mu \mathrm{M}$ had little effect on the radiation sensitivity compared to the untreated control samples. Decreased survival following irradiation in the celastroltreated cells was apparent in the $1 \mathrm{~Gy}$ irradiated cells and became more prominent at $4 \mathrm{~Gy}$. By contrast, treatment of the same lung cancer cell line with TG had very little effect on the cell survival following ionizing radiation (Fig. 2D). These results indicate that the drugs, which belong to the same triterpene family, have different effects on radiation sensitivity. Moreover the results suggest that the drugs may display differential effects on target molecules that determine radiation sensitivity. Thus, we investigated target molecules that may determine the radiation sensitivity upon drug treatment. Among the potential target molecules for radiation sensitivity in the cells, we examined EGFR, ErbB2, Akt and survivin since their decreased levels of expression are correlated with enhanced radiation sensitivity $(14,15)$.
Celastrol treatment of the cells significantly decreased levels of EGFR, ErbB2, and survivin, whereas, levels of the Akt protein were unaffected by the treatment (Fig. 3A). The decrease in the proteins was first observed at $0.1 \mu \mathrm{M}$ celastrol treatment and became prominent at $0.5 \mu \mathrm{M}$ at which concentration increased radiation sensitivity was also observed (Fig. 2C). Unlike celastrol, another triterpene, TG, had little effect on the levels of the proteins (Fig. 3B). Levels of EGFR, ErbB2, Akt, and survivin remained constant with all TG concentrations used (Fig. 3B). Constant levels of the radiation sensitivity modulators were also correlated with little or no effect on radiation sensitivity (Fig. 2D). Taken together, EGFR, ErbB2 and survivin, are potential target molecules of the celastrol-induced enhanced radiation sensitivity. Furthermore, the expression levels of the molecules are correlated with survival following the combined treatment with ionizing radiation and celastrol. In addition, the results demonstrate that the effect of celastrol is specific in that a structurally similar triterpene, TG had no effect on the response.

Since the effect of celastrol on the cellular radiation response is distinct from that of $\mathrm{TG}$, investigation of the responsible mechanism may uncover the cause of the differential drug effect on radiation sensitivity. We observed that the target proteins of celastrol are Hsp90 client proteins. Inhibitors of Hsp90, such as 17-AAG and geldanamycin, inhibit ATP-binding to Hsp90, which in turn reduces Hsp90 
A

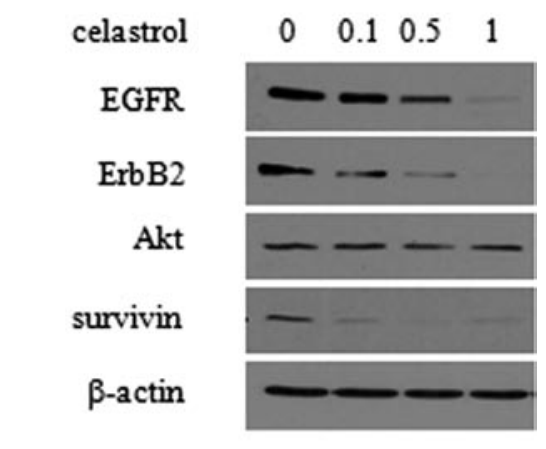

B

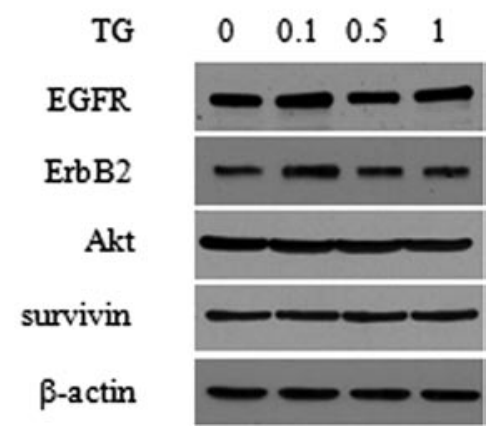

Figure 3. Western blotting of potential celastrol target proteins. NCI-H460 cells were treated for $24 \mathrm{~h}$ with $0,0.1,0.5$ or $1 \mu \mathrm{M}$ of celastrol (A) or TG (B). Levels of EGFR, ErbB2, Akt and survivin were detected by Western blot analysis. $\beta$-actin was detected as a control.

activity as a molecular chaperone and degrades certain Hsp90 client proteins. In order to prove that enhancement of radiation sensitivity by celastrol treatment is caused by inhibition of Hsp90, we examined the ATP-binding activity of Hsp90 in the presence or absence of celastrol (Fig. 4A). The ATP-pull down assay with whole cell extracts prepared from either celastrol- or TG-treated cells revealed that celastroltreated cells have less Hsp90 ATP-binding activity, whereas TG-treated and untreated control cells showed comparable levels of ATP-binding (Fig. 4A). The results suggest that celastrol acts as an inhibitor of Hsp90 by interfering with its ATP-binding, whereas TG does not. Since dissociation and degradation of certain client proteins of Hsp90 is another feature of Hsp90 inhibition, we examined these Hsp90induced effects on EGFR (Fig. 4B). Whereas TG treatment had little effect on the Hsp90-EGFR interaction, celastrol treatment dissociated EGFR from Hsp90, as expected from Hsp90 inhibition (Fig. 4B). Since degradation of Hsp90 client proteins following inhibition of Hsp90 is mediated by the proteasomal degradation pathway, we examined whether the degradation of EGFR is mediated by the proteasome (Fig. 4C). Addition of celastrol alone decreased the levels of EGFR whereas combined treatment with ALLN, an inhibitor of the proteasome and celastrol, maintained EGFR at levels comparable to that of untreated control. Therefore, celastrol treatment induces EGFR destabilization via the proteasomal degradation pathway. Taken together, celastrol inhibits Hsp90 by interfering with its ATP-binding and causes dissociation
$\mathbf{A}$

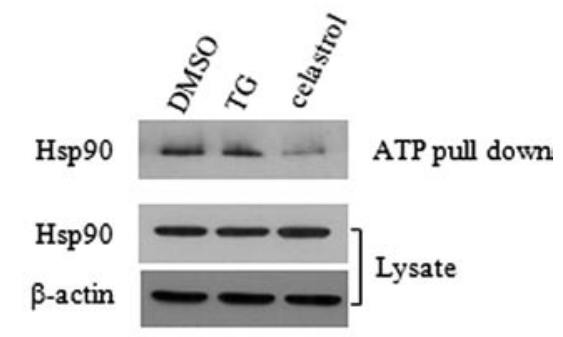

B

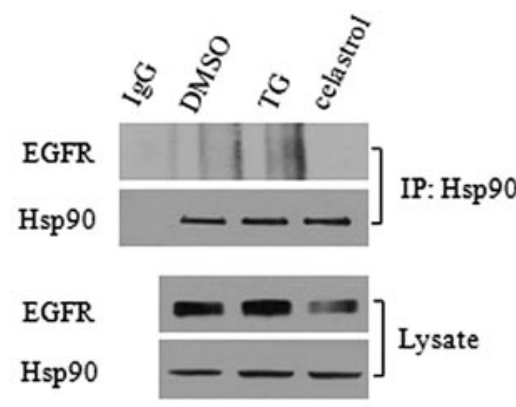

C

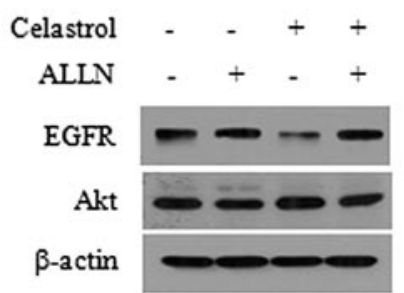

Figure 4. Inhibition of Hsp90 by celastrol. (A) Inhibition of the ATP-binding activity of Hsp90. ATP-pull down assay was performed using cell extracts prepared from either celastrol or TG-treated cells. Levels of ATP-bound Hsp90 were determined by Western blot analysis. Total levels of Hsp90 in the cell lysate were also detected. $\beta$-actin was detected as a control. (B) Dissociation of the Hsp90 client EGFR from Hsp90 following celastrol treatment. Immunoprecipitation was carried out with cell extracts that were prepared from celastrol or TG-treated cells. Levels of EGFR and Hsp90 were determined by Western blot analysis. DMSO treatment was used as a control. Total levels of EGFR and Hsp90 in the cell lysate were also determined by Western blot analysis (bottom panel). (C) Proteasomal degradation of EGFR following celastrol treatment. Cells were treated with celastrol $(0.5 \mu \mathrm{M})$ in the presence or absence of the proteasome inhibitor, $\operatorname{ALLN}(50 \mu \mathrm{M})$. Levels of EGFR and Akt were determined by Western blot analysis. $\beta$-actin was used as a control.

and subsequent degradation of certain client protein such as EGFR via the proteasomal pathway.

Since radiation sensitivity is also determined by factors involved in cell growth arrest or death, we examined the effect of celastrol on p53 as it is one of the main determinants of radiation sensitivity. Celastrol treatment of NCI-H460 cells increased $\mathrm{p} 53$ level (Fig. 5A). The increase could be seen from $6 \mathrm{~h}$ and on with $1 \mu \mathrm{M}$ celastrol. As p53 phosphorylation at serine residues 15 and 20 is responsible for the p53 increase, we assessed these phosphorylations. Phosphorylation at p53 Ser15 and Ser20 was increased by celastrol treatment (Fig. 5A). The p53 responses by celastrol treatment mimicked the cellular radiation responses, whereby irradiation with ionizing radiation increased p53 phosphorylation and its protein level (right half of Fig. 5A). In line with the increased phosphorylation, celastrol treatment caused dissociation of MDM2 from p53 so that MDM2 is unable to ubiquitinate p53 (Fig. 5B). 
A

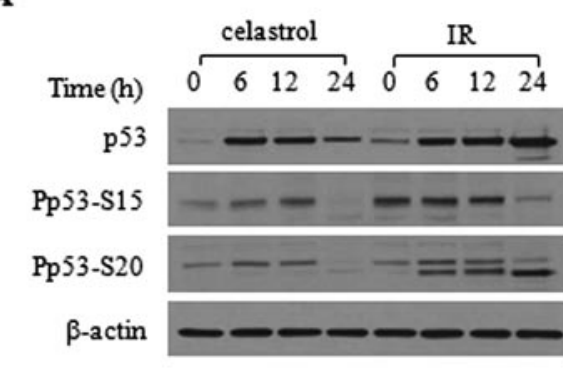

C

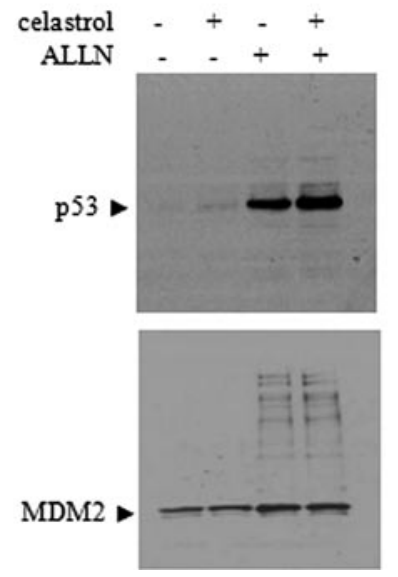

B

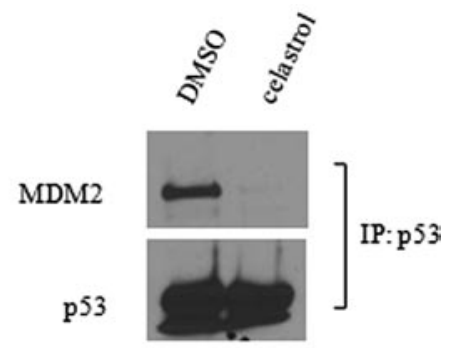

D

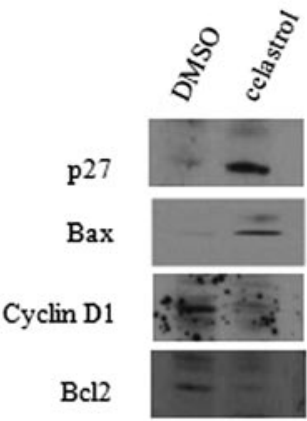

Figure 5. Effect of celastrol treatment on p53. (A) Effect on p53 phosphorylation following celastrol treatment. p53 phosphorylation was determined by Western blot analysis. Cells were incubated with celastrol $(1 \mu \mathrm{M})$ for $0,6,12$ and $24 \mathrm{~h}$. For comparison, extracts prepared from 5 Gy irradiated cells were used to detect p53 and phosphorylated p53. $\beta$-actin was used as a control. (B) Dissociation of p53 from MDM2 following celastrol treatment. Immunoprecipitation was carried out to examine the effect of celastrol on the interaction between p53 and MDM2. Immunoprecipiation was performed with a p53 antibody and the Western blot detection with antibodies against MDM2 or p53. Cell extracts for immunoprecipitation were prepared from celatsrol-treated or non-treated samples. (C) Effect of combined treatment with celastrol and the proteasome inhibitor on p53 and MDM2. Levels of p53 and MDM2 were determined by Western blot analysis. NCI-H460 cells were treated with or without ALLN (50 $\mu \mathrm{M})$ and celastrol (1 $\mu \mathrm{M})$. (D) Effect of celastrol on the regulators of cell cycle or of cell death. Cells were treated with celastrol and the protein levels were determined by Western blot analysis.

Treatment of cells with celastrol alone slightly increased p53 whereas co-treatment with ALLN further increased the p53 levels (Fig. 5C). Considering the fact that celastrol inhibits proteasomal activity, co-treatment of cells with celastrol and proteasome inhibitor might potentiate cell killing by further increasing p53 levels. Celastrol treatment of cells increased Bax, one of the p53 target genes, and a pro-apoptotic protein, while it repressed $\mathrm{Bcl} 2$ expression, an anti-apoptotic protein (Fig. 5D). Cyclin D1 was decreased by celastrol treatment while p27 was increased. Thus, it seems that celastrol treatment increased molecules that are involved in either cell death or growth arrest, while at the same time it decreased the levels of proteins which are necessary for survival or cell cycle progression. Taken together, celastrol-induced changes in the levels of EGFR, ErbB2, survivin and p53 might be responsible for the enhancement of radiation sensitivity.

\section{Discussion}

In the present study, we examined the radiosensitizing activity of celastrol. Celastrol enhanced the radiation sensitivity of a human lung cancer cell line, NCI-H460, by utilizing various mechanisms.

First, celastrol treatment destabilized certain Hsp90 client proteins which also serve as regulators of radiation sensitivity. Radiomodulatory factors, such as EGFR, ErbB2 and survivin, are Hsp90 client proteins (2). When client proteins are destabilized following treatment with Hsp90 inhibitors, radiation sensitivity is increased. For example, combined treatment of cancer cells with the Hsp90 inhibitor, 17-AAG, and ionizing radiation enhances the radiation sensitivity of various cancer cells (14).

Celastrol inhibited Hsp90's ATP-binding activity and dissociated a typical client protein, EGFR, resulting in destabilization of the client protein (Fig. 4). As many of the Hsp90 client proteins including EGFR are involved in the modulation of radiation responses, enhancement of radiation sensitivity by celastrol is probably due to inhibition of Hsp90. Celastrol's activity in the radiation response seems to require a specific functional moiety, because a structurally similar compound TG could not exert the same kind of responses as celastrol. Thus, isolation or synthesis of derivatives of celastrol might lead to the development of a more effective radiosensitizer or anti-cancer compound.

Celastrol has been investigated for its anti-inflammatory and anticancer activity $(10,16)$. Celastrol inhibits the activation of NF- $\kappa \mathrm{B}$ through targeting I $\mathrm{B}$ kinase and potentiates TNF-induced apoptosis by inhibiting NF- $\kappa \mathrm{B}$-regulated gene expression (4). Since NF- $\kappa \mathrm{B}$ activity is critical for cell survival following ionizing radiation (17), inhibition of $N F-\kappa B$ by celastrol treatment might have contributed to the enhancement of radiation sensitivity. 
According to previous reports, inhibition of DNA damage repair as well as degradation of proliferation signaling molecules underlie the radiosensitizing effect of Hsp90 inhibitors $(14,18)$. Since we identified the celastrol-induced degradation of proteins involved in proliferation, it was of interest to examine whether celastrol also inhibits the activity of DNA damage response factors, similarly to 17-AAG (18).

p53 regulation was also shown to contribute to the radiosensitizing effects of celastrol. p53 regulates radiosensitivity by modulating the transcription of many responsive genes involved in either growth arrest (such as $\mathrm{p} 21^{\mathrm{WAFI}}$ ) or apoptosis (such as bax, puma and noxa) (19). Celastrol increased p53 by increasing phosphorylation of Ser15 and Ser20 residues (Fig. 5A). p53 phosphorylation at Ser15 interrupts the binding of MDM2 with p53 and protects p53 from degradation (20). Since DNA damage induces p53 Ser15 phosphorylation, it is of interest to investigate whether celastrol treatment brings about cellular DNA damage.

In addition to p53 phosphorylation, inhibition of the proteasome seems to be involved in the increased p53 activity by celastrol. Unlike other Hsp90 inhibitors, celastrol exhibits additional activity as a potent proteasome inhibitor (10). Thus, increased p53 is probably due to both phosphorylation at key residues and inhibition of proteasomal degradation, which together contribute to the enhanced radiation sensitivity.

Our results have shown that celastrol treatment increases the expression of Bax, a p53 target gene involved in apoptosis (Fig. 5D). At the same time, the expression of the anti-apoptotic protein, $\mathrm{Bcl} 2$, was decreased by celastrol treatment, suggesting that the cellular environment favors cell death following the combined treatment with radiation and celastrol. As the investigation was carried out with the NCI-H460 cells, which express wild-type $\mathrm{p} 53$, increased $\mathrm{p} 53$ levels following celastrol treatment may have sensitized the cells to ionizing radiation because ionizing radiation-induced cell death of NCI-H460 cells is dependent upon p53 activity (21). However, celastrol's radiosensitizing effect may not be limited to cells with wildtype p53. A recent report demonstrates that celastrol sensitized the p53-null prostate cancer cell line, PC-3 (22). Our results also support the notion that celastrol treatment triggers degradation of key molecules involved in proliferation signaling, such as EGFR (Fig. 4). Multiple activities of celastrol in combination with radiation may be of use in treating many types of cells regardless of the p53 status.

Comparison of the results with two structurally similar compounds, celastrol and TG suggests that a specific functional moiety might be required for enhanced radiosensitivity. It further suggests that other triterpenoids might exhibit a similar radiosensitizing effect. Further analysis with other purified triterpenoids should resolve these issues and aid in the development of improved tumor control when the drugs are combined with ionizing radiation treatment.

\section{Acknowledgements}

This study was supported by the Nuclear Research and Development program of the National Research Foundation of Korea funded by the Korea government (MEST grant code: 2010-0005406).

\section{References}

1. Tofilon PJ and Camphausen K: Molecular targets for tumor radiosensitization. Chem Rev 109: 2974-2988, 2009.

2. Camphausen K and Tofilon PJ: Inhibition of Hsp90: a multitarget approach to radiosensitization. Clin Cancer Res 13: 4326-4330, 2007.

3. Salminen A, Lehtonen M, Paimela T and Kaarniranta K: Celastrol: molecular targets of Thunder God Vine. Biochem Biophys Res Commun 394: 439-442, 2010.

4. Sethi G, Ahn KS, Pandey MK and Aggarwal BB: Celastrol, a novel triterpene, potentiates TNF-induced apoptosis and suppresses invasion of tumor cells by inhibiting NF-kappaBregulated gene products and TAK1-mediated NF-kappaB activation. Blood 109: 2727-2735, 2007.

5. Lee JH, Koo TH, Yoon H, Jung HS, Jin HZ, Lee K, Hong YS and Lee JJ: Inhibition of NF-kappa B activation through targeting I kappa B kinase by celastrol, a quinone methide triterpenoid. Biochem Pharmacol 72: 1311-1321, 2006.

6. Zhu H, Ding WJ, Wu R, Weng QJ, Lou JS, Jin RJ, Lu W, Yang B and $\mathrm{He}$ QJ: Synergistic anti-cancer activity by the combination of TRAIL/APO-2L and celastrol. Cancer Invest 28: 23-32, 2010.

7. Zhang T, Li Y, Yu Y,Zou P, Jiang Y and Sun D: Characterization of celastrol to inhibit hsp90 and cdc37 interaction. J Biol Chem 284: 35381-35389, 2009.

8. Sharp S and Workman P: Inhibitors of the HSP90 molecular chaperone: current status. Adv Cancer Res 95: 323-348, 2006.

9. Zhang T, Hamza A, Cao X, Wang B, Yu S, Zhan CG and Sun D: A novel Hsp90 inhibitor to disrupt Hsp90/Cdc37 complex against pancreatic cancer cells. Mol Cancer Ther 7: 162-170, 2008.

10. Yang H, Chen D, Cui QC, Yuan X and Dou QP: Celastrol, a triterpene extracted from the Chinese 'Thunder of God Vine', is a potent proteasome inhibitor and suppresses human prostate cancer growth in nude mice. Cancer Res 66: 4758-4765, 2006.

11. Ngassapa O, Soejarto DD, Pezzuto JM and Farnsworth NR: Quinone-methide triterpenes and salaspermic acid from Kokoona ochracea. J Nat Prod 57: 1-8, 1994.

12. Chung HJ, Yoon SI, Shin SH, Koh YA, Lee SJ, Lee YS and Bae S: p53-Mediated enhancement of radiosensitivity by selenophosphate synthetase 1 overexpression. J Cell Physiol 209: 131-141, 2006.

13. Park MT, Choi JA, Kim MJ, Um HD, Bae S, Kang CM, Cho CK, Kang S, Chung HY, Lee YS and Lee SJ: Suppression of extracellular signal-related kinase and activation of p38 MAPK are two critical events leading to caspase-8- and mitochondriamediated cell death in phytosphingosine-treated human cancer cells. J Biol Chem 278: 50624-50634, 2003.

14. Russell JS, Burgan W, Oswald KA, Camphausen K and Tofilon PJ: Enhanced cell killing induced by the combination of radiation and the heat shock protein 90 inhibitor 17-allylamino-17-demethoxygeldanamycin: a multitarget approach to radiosensitization. Clin Cancer Res 9: 3749-3755, 2003.

15. Lu B, Mu Y, Cao C, Zeng F, Schneider S, Tan J, Price J, Chen J, Freeman M and Hallahan DE: Survivin as a therapeutic target for radiation sensitization in lung cancer. Cancer Res 64: 2840-2845, 2004.

16. Petronelli A, Pannitteri G and Testa U: Triterpenoids as new promising anticancer drugs. Anticancer Drugs 20: 880-892, 2009.

17. Sharma V, Hupp CD and Tepe JJ: Enhancement of chemotherapeutic efficacy by small molecule inhibition of NF-kappaB and checkpoint kinases. Curr Med Chem 14: 1061-1074, 2007.

18. Dote H, Burgan WE, Camphausen K and Tofilon PJ: Inhibition of hsp90 compromises the DNA damage response to radiation. Cancer Res 66: 9211-9220, 2006.

19. Gudkov AV and Komarova EA: The role of p53 in determining sensitivity to radiotherapy. Nat Rev Cancer 3: 117-129, 2003.

20. Bode AM and Dong Z: Post-translational modification of p53 in tumorigenesis. Nat Rev Cancer 4: 793-805, 2004.

21. Zhang D, Zaugg K, Mak TW and Elledge SJ: A role for the deubiquitinating enzyme USP28 in control of the DNA-damage response. Cell 126: 529-542, 2006

22. Dai Y, DeSano JT, Meng Y, Ji Q, Ljungman M, Lawrence TS and $\mathrm{Xu} \mathrm{L}$ : Celastrol potentiates radiotherapy by impairment of DNA damage processing in human prostate cancer. Int $\mathbf{J}$ Radiat Oncol Biol Phys 74: 1217-1225, 2009. 